\title{
Ovarian, Fallopian Tube, and Primary Peritoneal Carcinoma pM1a TNM Finding v8
}

National Cancer Institute

\section{Source}

National Cancer Institute. Ovarian, Fallopian Tube, and Primary Peritoneal Carcinoma

pM1a TNM Finding v8. NCI Thesaurus. Code C139937.

Ovarian, fallopian tube, or primary peritoneal carcinoma with pleural effusion with positive cytology. (from AJCC 8th Ed.) 